\title{
Erratum to: Polymorphism of rs3737597 in DISC1 Gene on Chromosome 1q42.2 in sALS Patients: a Chinese Han Population Case-Control Study
}

\author{
Libin Deng ${ }^{2,3} \cdot$ Liwei Huo ${ }^{4}$ Jie Zhang ${ }^{1,3} \cdot$ Xiaoli Tang ${ }^{3} \cdot$ Zhujun Cheng $^{2} \cdot$ Gang $\mathrm{Li}^{3}$ • \\ Xin Fang ${ }^{1} \cdot$ Jinsong $\mathrm{Xu}^{5} \cdot$ Xiong $\mathrm{Zhang}^{6} \cdot$ Renshi Xu $^{1}$
}

Published online: 4 May 2016

(C) Springer Science+Business Media New York 2016

\section{Erratum to: Mol Neurobiol}

DOI: 10.1007/s12035-016-9869-3

The authors of this paper unfortunately made a mistake on the author group. The Family name of Dr. Liwei Huo on the proof is incorrect.

With this, the authors do hereby publish this correction note to correct "Liwei Hou" to "Liwei Huo".

The online version of the original article can be found at http://dx.doi.org/ 10.1007/s12035-016-9869-3.

\author{
Xiong Zhang \\ xiong715@126.com \\ 13767015770@163.com; xurenshi@yahoo.com
}

$\triangle$ Renshi Xu

1 Department of Neurology, The First Affiliated Hospital of Nanchang University, Nanchang 330006, Jiangxi, China

2 Institute of Translational Medicine, Nanchang University, Nanchang 330031, Jiangxi, China

3 College of Basic Medical Science, Nanchang University, Nanchang 330006, Jiangxi, China

4 School of Public Health, Nanchang University, Nanchang 330006, Jiangxi, China

5 Deparment of Vasculocardiology, The Second Affiliated Hospital of Nanchang University, Nanchang 330006, Jiangxi, China

6 Department of Neurology, Guangdong General Hospital, Guangdong Academy of Medical Sciences, Guangdong Neuroscience Institute, Guangzhou 510080, China 